\title{
Shifts in the Skin Microbiota after UVB Treatment in Adult Atopic Dermatitis
}

\author{
Astrid Haaskjold Lossius ${ }^{a, b}, c$ Olav Sundnes ${ }^{a, b}, c$ Anna Cäcilia Ingham ${ }^{d}$ \\ Sofie Marie Edslev ${ }^{d}$ Jørgen Vildershøj Bjørnholt ${ }^{a, e}$ Berit Lilje $^{d}$ \\ Maria Bradley ${ }^{f}$ Samina Asad ${ }^{f}$ Guttorm Haraldsen ${ }^{a, c}$ Paal Skytt-Andersen ${ }^{d}$ \\ Jan-Øivind Holm ${ }^{a}$, b Teresa Løvold Berents ${ }^{\text {b, } g}$ \\ ${ }^{a}$ Institute of Clinical Medicine, University of Oslo, Oslo, Norway; ${ }^{b}$ Department of Dermatology, Oslo University

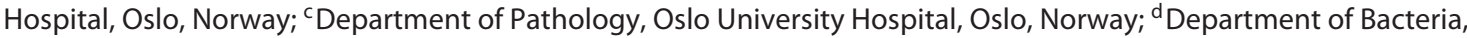 \\ Parasites, and Fungi, Statens Serum Institut, Copenhagen, Denmark; ${ }^{e}$ Department of Microbiology, Oslo University \\ Hospital, Oslo, Norway; 'Division of Dermatology and Venereology, Department of Medicine, Karolinska Institutet, \\ Stockholm, Sweden; ${ }^{9}$ Regional Unit of Asthma, Allergy and Hypersensitivity, Oslo University Hospital, Oslo, Norway
}

\section{Keywords}

Atopic dermatitis · Phototherapy · Skin microbiota

\section{Abstract}

Background: The pathophysiology in atopic dermatitis (AD) is not fully understood, but immune dysfunction, skin barrier defects, and alterations of the skin microbiota are thought to play important roles. AD skin is frequently colonized with Staphylococcus aureus (S. aureus) and microbial diversity on lesional skin (LS) is reduced compared to on healthy skin. Treatment with narrow-band ultraviolet $B$ (nb-UVB) leads to clinical improvement of the eczema and reduced abundance of S. aureus. However, in-depth knowledge of the temporal dynamics of the skin microbiota in $A D$ in response to nb-UVB treatment is lacking and could provide important clues to decipher whether the microbial changes are primary drivers of the disease, or secondary to the inflammatory process. Objectives: To map the temporal shifts in the microbiota of the skin, nose, and throat in adult AD patients after nb-UVB treatment. Methods: Skin swabs were taken from

karger@karger.com www.karger.com/drm

Karger $\stackrel{\text { ! }}{\div}$
(C) 2021 The Author(s)

Published by S. Karger AG, Basel

This is an Open Access article licensed under the Creative Common Attribution-NonCommercial-4.0 International License (CC BY-NC) (http://www.karger.com/Services/OpenAccessLicense), applicable to the online version of the article only. Usage and distribution for commercial purposes requires written permission. lesional AD skin $(n=16)$ before and after 3 treatments of $n b$ UVB, and after $6-8$ weeks of full-body treatment. We also obtained samples from non-lesional skin (NLS) and from the nose and throat. All samples were characterized by $16 \mathrm{~S}$ rRNA gene sequencing. Results: We observed shifts towards higher diversity in the microbiota of lesional AD skin after 6-8 weeks of treatment, while the microbiota of NLS and of the nose/throat remained unchanged. After only 3 treatments with nb-UVB, there were no significant changes in the microbiota. Conclusion: Nb-UVB induces changes in the skin microbiota towards higher diversity, but the microbiota of the nose and throat are not altered. @ 2021 The Author(s)

Published by S. Karger AG, Basel

\section{Introduction}

Atopic dermatitis (AD) is the most prevalent inflammatory skin disease, with a broad impact on patients' quality of life and on the health care system $[1,2]$. It is characterized by itchy, red, scaly skin and increased risk 
of skin infections [3]. The pathogenesis is complex, and immune dysfunction, skin barrier defects, and alterations in the skin microbiota are thought to play important roles $[2,4,5]$. Whether the microbial abnormalities are primary drivers of $\mathrm{AD}$ or whether they are secondary events resulting from inflammation or skin barrier dysfunction is still not fully elucidated.

Colonization of the skin with Staphylococcus aureus ( $S$. aureus) is more frequent in patients with $\mathrm{AD}[6,7]$, and the abundance of $S$. aureus correlates with disease severity [7-9]. This is partly explained by $S$. aureus thriving better in the higher $\mathrm{pH}$ conditions of $\mathrm{AD}$ skin than in the more acidic environment of healthy skin [10]. In AD skin, there is an increased relative abundance of $S$. aureus and S. epidermidis, while on the genus level, Cutibacterium, Streptococcus, Acinetobacter, Corynebacterium, and Prevotella are decreased $[11,12]$.

During eczema flares, the lesional skin (LS) microbiota becomes less diverse, but after weeks of adequate therapy, it seems to approximate diversity levels comparable to baseline [13-19]. A recent review emphasized the inconsistent findings on bacterial diversity on LS versus non-lesional skin (NLS) in AD [20]. While most studies report lower diversity on LS than NLS [12, 21, 22], some report no differences [23, 24].

Interestingly, the nasal microbiota in $\mathrm{AD}$ patients is more uniform than in healthy controls [23], and the microbial composition in the nose correlates to $\mathrm{AD}$ severity in children with increased abundance of Staphylococcus spp. in more severe AD [25, 26].

Phototherapy with narrow-band ultraviolet B (nbUVB) exerts anti-bacterial effects [27-29] and alters the levels of the anti-microbial peptides (AMPs) defensins, S100-proteins, cathelicidin, and ribonuclease 7 [30-32]. Previous studies have shown, by culture-based methods, that UVB treatment decreases the relative abundance of S. aureus in AD skin in adults and children [33-35]. However, conventional culturing fails to grow $80 \%$ of bacterial species, and applying culture-independent methods like 16S rRNA gene sequencing means the whole bacterial community can be characterized [36, 37].

We aimed to map the changes in the skin, nose, and throat microbiota with $16 \mathrm{~S}$ rRNA gene sequencing before and after 6-8 weeks of full-body nb-UVB treatment in adult patients with $\mathrm{AD}$. We also wanted to explore whether only 3 treatments of nb-UVB could induce any shifts in the skin microbiota before signs of clinical remission were evident.

\section{Materials and Methods}

\section{Patients}

Adults ( $n=16)$ with $\mathrm{AD}$, according to the criteria of Hanifin and Rajka [38], were recruited to the study and the same cohort has been described previously [39]. Prior to inclusion, systemic antibiotics and immunosuppressive therapy were avoided for 4 weeks. Topical immunosuppressive therapy (corticosteroids and calcineurin inhibitors) and topical anti-bacterial therapy were avoided for 2 weeks. Structured interviews were performed and disease severity and morbidity were assessed with validated scoring tools: the Eczema Area and Severity Index (EASI) [40], the SCORing Atopic Dermatitis (SCORAD) index [41], the PatientOriented Eczema Measure (POEM) [42], and the Dermatology Life Quality Index (DLQI) [43]. Saliva was collected in Oragene ${ }^{\mathrm{TM}}$ DNA saliva sampling kits (DNA Genotek, Ottawa, ON, Canada) and tested for the 3 most common mutations in the genes encoding filaggrin (FLG): R501X, R2447X, and 2282del4. Serum samples, to measure IgE, eosinophils, and vitamin D levels, and microbial samples were collected.

\section{Intervention}

The nb-UVB minimal erythema dose [44] was established by Dermalight $^{\circledR} 80$ MED-tester (Dr Hönle, Medizintechnik, Gilching, Germany) and 1 lesion with active $\mathrm{AD}$ was chosen, primarily the antecubital crease. This lesion was irradiated with $1 \mathrm{MED}$ on days 0,2 , and 4 with the small, hand-held nb-UVB device, Dermalight ${ }^{\circledR} 80$. Subsequently, the patients underwent full-body nb-UVB 3 times a week for 6-8 weeks, with incremental dosages, with a total of 12-25 treatments. Clinical severity scores (EASI, SCORAD, POEM, and DLQI) were registered before treatment (day 0), after 3 treatments (day 7), and after 6-8 weeks of treatment.

\section{Microbial Sampling}

Microbial samples were collected at 3 time points: on days 0 and 7 (before and after local short-term nb-UVB treatment on days 0,2 , and 4) and after full-body treatment (3 times/week for 6-8 weeks). Samples were collected by rubbing a dry ESwab (Copan, Brescia, Italy) against an area of $5 \mathrm{~cm}^{2}$ of LS and NLS for 30 s. LS samples were primarily taken from the antecubital crease, while NLS skin was sampled from the nates, regarded as UV-naïve skin before treatment. Nose samples were obtained by rotating a dry Eswab in both anterior nares and the throat samples by rubbing the fauces. All samples were immediately frozen and stored at $-80^{\circ} \mathrm{C}$.

\section{DNA Extraction and Amplicon Sequencing}

Bacterial DNA was isolated from Eswab after enzymatic prelysis by mixing $200 \mu \mathrm{L}$ sample with $50 \mu \mathrm{L}$ enzymatic TE lysis buffer (lysostaphine [SAE0091] 2,5U, mutanolysin [sae0092] 25U, lysozyme [L4919] $3 \mathrm{mg}$ [Sigma-Aldrich, St. Louis, USA]) for $30 \mathrm{~min}$ at $37^{\circ} \mathrm{C}$, adding $20 \mu \mathrm{L}$ proteinase K (RPROTKSOL-RO, Sigma-Aldrich), and incubated at $56^{\circ} \mathrm{C}$ for $30 \mathrm{~min} .200 \mu \mathrm{L}$ was extracted on a MagNA Pure 96 system (Roche, Mannheim, Germany) with a DNA and Viral NA small volume kit (Roche). Previously evaluated primers (341F: $5^{\prime}$-CCTACGGGNGGCWGCAG-3'; 805R: $5^{\prime}$-GACTACHVGGGTATCTAATCC-3'), preceded by heterogeneity spacers, were used for amplification of the $\mathrm{V} 3-\mathrm{V} 4$ region of the $16 \mathrm{~S}$ rRNA gene [45]. Library construction and sequencing was 
performed on Illumina MiSeq (Illumina Inc., San Diego, USA), using a 600 cycle V3 kit. To resolve species-level affiliations within the genus Staphylococcus, we implemented a tuf-gene sequencing approach as previously described [46].

\section{Pre-Processing of Sequencing Data}

Raw reads were demultiplexed using the bcl2fastq conversion software (Illumina, San Diego, CA, USA). Heterogeneity spacers and primers were trimmed off at an $8 \%$ error rate (1 mismatch per primer sequence) with cutadapt (v2.3) of $16 \mathrm{~S}$ rRNA and tuf gene reads [47]. Both primers had to be detected in the respective reads to retain a read pair. We used the $R$ package dada2 (v1.12.1) for amplicon sequence variant (ASV) inference from trimmed reads [48]. Except for the truncation lengths (Appendix 1), the dada2 pipeline was used with default settings. Consensus removal of chimeras was performed. Samples with a read count $<5,000$ after quality filtering were re-sequenced. ASVs resulting from $16 \mathrm{~S}$ rRNA gene sequences were taxonomically classified with dada2's assignTaxonomy() and addSpecies() functions, using the Silva reference database and species-level training set (v132), respectively [49]. Staphylococcal ASVs from $t u f$ gene sequences were classified with the assignTaxonomy() function, based on the taxonomic database designed by Iversen et al. [46].

The ASV count tables and taxonomic table were integrated using the $R$ package phyloseq [50]. The 16S rRNA gene-derived count data of skin, nose, and throat samples was separately subjected to contaminant identification removal using the $R$ package decontam [51] and manually contaminant removal (Appendix 2). One skin sample with $<4,500$ reads after merging was excluded from downstream analysis.

\section{Statistical Analysis}

Statistical analyses were performed in $R$ v3.6.0 [52] with the packages phyloseq and its dependencies, vegan, cluster, factoextra, markovchain, and lmerTest [50, 53-58]. Visualizations were generated with ggplot2, diagram, and ComplexHeatmap [59-61]. For each sample, bacterial alpha diversity, the diversity within samples, and measurements of both species' richness and evenness, were calculated on raw counts by the inverse Simpson index. Alpha diversity on LS and NLS before treatment was compared using a paired Wilcoxon signed-rank test. Alpha diversity before treatment, and after local short-term and full-body treatment for 6-8 weeks was compared by paired Wilcoxon signed-rank tests. Correlation between the number of nb-UVB sessions and change in bacterial diversity was calculated by Spearman's rank correlation. All presented taxonomic barplots are based on relative abundance.

The count data was Hellinger-transformed, i.e., sample-wise proportions were calculated, and subsequently square root-transformed. Principal co-ordinates analysis (PCoA) based on the BrayCurtis distance was performed to visualize differences in the bacterial community structure over time. Samples were grouped into community state types (CSTs) by partitioning around medoid (PAM) clustering, based on the Jensen-Shannon distance. A consensus decision about pre-determining the optimal number of clusters was made by means of the gap statistic, silhouette width, and the elbow method. CST dynamics were visualized as Markov chains showing transition probabilities between CSTs over time.

We performed canonical correspondence analysis (CCpnA), a multivariate constrained ordination method, to model bidirec-
Table 1. Adults with atopic dermatitis $(n=16)$

\begin{tabular}{lc}
\hline Median age, years (range) & $25.5(20-73)$ \\
Sex & 11 \\
$\quad$ Female & 5 \\
$\quad$ Male & \\
Filaggrin mutation & a \\
$\quad$ WT & 12 \\
$\quad$ Mutation & 4 \\
Serum IgE $^{\mathrm{b}}$ & \\
$\quad$ Elevated & 8 \\
$\quad$ Normal & 8 \\
Eosinophil count & \\
$\quad$ Elevated & 2 \\
$\quad$ Normal & 10 \\
$\quad$ Not available & 4 \\
Vitamin D supplementation & \\
$\quad$ Yes & 9 \\
$\quad$ No & 7 \\
Median number of nb-UVB sessions (range) & $20(12-25)$ \\
\hline
\end{tabular}

Values express $n$, unless otherwise indicated. Serum was sampled from all patients prior to nb-UVB treatment for the measurement of IgE and eosinophils. nb-UVB, narrow-band ultraviolet B; WT, wild type.

a Saliva was genotyped for the most common mutations in the genes encoding filaggrin: R501X, R2447X, and 2282del4. Patients with the R501X mutation were heterozygous; 1 patient was compound heterozygous (2 different mutations: R501X/R2447X).

${ }^{\mathrm{b}}$ Reference range $0-114 \mathrm{kU} / \mathrm{L}$.

${ }^{\mathrm{c}}$ Reference range $<0.4 \times 10^{9} / \mathrm{L}$.

Table 2. Vitamin D levels and clinical severity scores before and after treatment

\begin{tabular}{lcc}
\hline & Before treatment & After treatment \\
\hline Serum vitamin $D^{\text {a }}$ levels & $44-116(81.1)$ & $67-174(123.4)$ \\
EASI & $3.6-14.7(9.1)$ & $0-24.3(5.1)$ \\
SCORAD & $22.5-65(40.8)$ & $5-69.5(27.2)$ \\
POEM & $8-23(16.6)$ & $4-23(10.6)$ \\
DLQI & $5-22(11.2)$ & $1-11(5.3)$ \\
\hline
\end{tabular}

Values express range (mean). EASI, Eczema Activity and Severity Index; SCORAD, SCORing Atopic Dermatitis index; POEM, Patient-Oriented Eczema Measure; DLQI, Dermatology Life Quality Index.

a 25-OH-vitamin D (reference range $37-131 \mathrm{nmol} / \mathrm{L}$ ).

tional relations between ASV abundances and CST affiliation, alpha diversity, and disease severity. The function $c c a()$ from the package vegan [53] was used.

The 16S rRNA gene and tuf gene sequences are available through the European Nucleotide Archive (ENA) at the European Bioinformatics Institute (EBI) under accession No. PRJEB41859. 


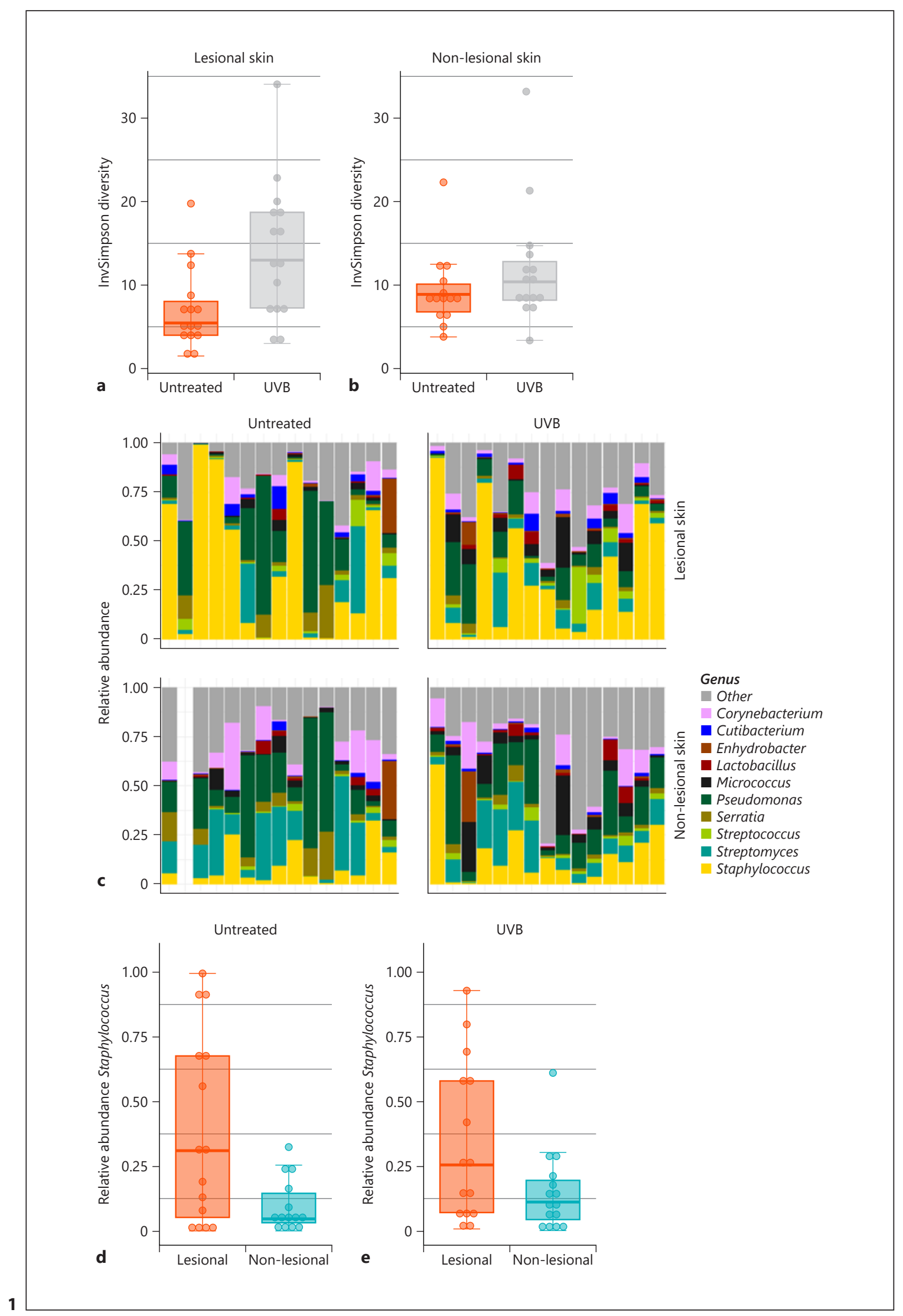

(For legend see next page.) 


\section{Results}

Adults with $\mathrm{AD}(n=16)$ were included ( 11 females and 5 males; median age 25.5 years [range $20-73$ years]) and clinical and demographic data are presented in Table 1. Four of the patients had a mutation in the gene encoding FLG (Table 1). At inclusion, most patients scored a moderate $\mathrm{AD}$ (Table 2 ), and after $6-8$ weeks of treatment there was a reduction in all the severity scores in most patients as previously described [39]. Vitamin D levels increased significantly (Table 2; online suppl. Fig. S1; see www. karger.com/doi/10.1159/000515236 for all online suppl. material).

\section{Bacterial Diversity Increases on LS after 6-8 Weeks of} $n b-U V B$

The bacterial alpha diversity increased on LS after 6-8 weeks of treatment with nb-UVB ( $p=0.013$; Fig. 1a). In contrast, the difference before and after treatment on NLS was not significant (Fig. 1b). We observed a trend of lower alpha diversity on LS than on NLS before treatment, although not significant (data not shown). There was no correlation between the number of nb-UVB sessions and change in bacterial diversity for LS (Spearman's rank correlation $\rho: 0.1320122, p=0.65$ ) or NLS (Spearman's rank correlation $\rho:-0.2702212, p=0.37$; data not shown).
The relative proportions of the different genera on LS compared to NLS before and after treatment are shown in Figure 1c; the plot shows greater abundance of Staphylococcus on LS than on NLS before and after treatment. The relative abundance of Staphylococcus was higher on LS than on NLS before treatment ( $p=0.001$, Fig. 1d). After treatment for 6-8 weeks, the relative abundance of Staphylococcus was still higher on LS ( $p=0.001$, Fig. 1e). We further did tuf-sequencing to explore whether the relative abundances of $S$. aureus and S. epidermidis changed after treatment, but we did not observe any significant changes after 6-8 weeks of treatment with nb-UVB (data not shown). Also, we did not find any significant changes in the abundance of Pseudomonadaceae (data not shown).

\section{Dynamics of the Bacterial Communities}

To investigate the dynamics of the bacterial community, we did clustering into CSTs, and 4 different CSTs were identified. CST 1 and CST 4 were mainly dominated by the family Staphylococcaceae. At the genus level, CST 1 and CST 4 were dominated by Staphylococcus, while CST 3 was dominated by Pseudomonas (Fig. 2). We implemented a CCpnA to model multivariate relationships between CST affiliation, alpha diversity, and clinical severity scores, which visually confirmed the clustering of the samples into 4 CSTs (Fig. 3). CSTs 1 and 4 were more

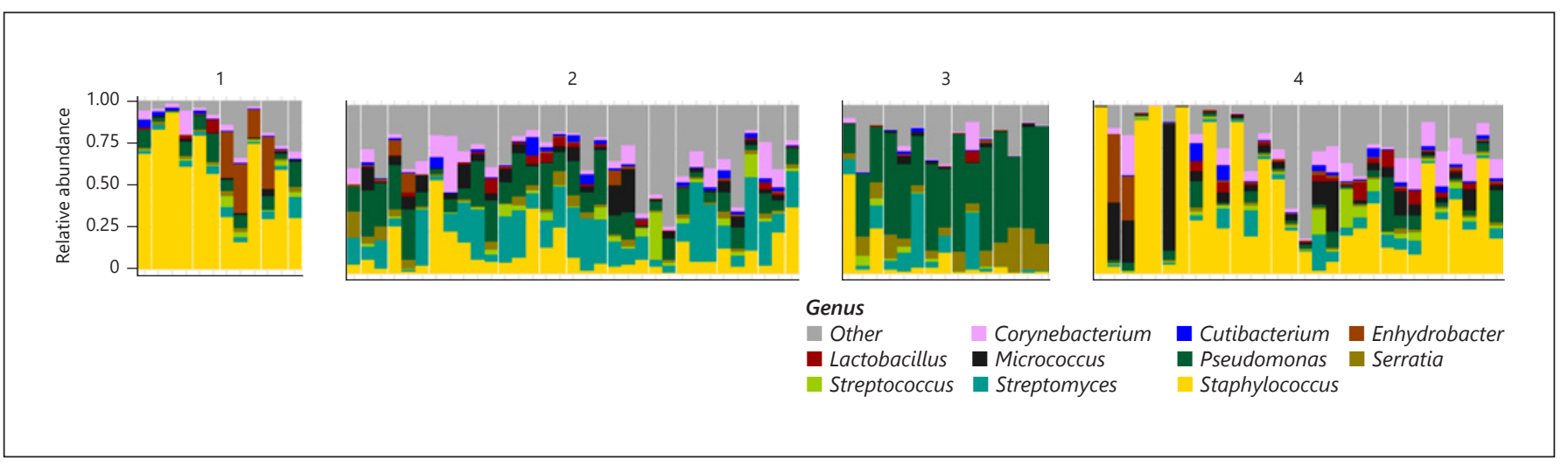

Fig. 2. Community state types (CSTs) in adults with atopic dermatitis. Four different CSTs were identified. At the genus level, CST 1 and CST 4 were dominated by Staphylococcus, while CST 3 was dominated by Pseudomonas. Each column represents 1 sample.

Fig. 1. Bacterial alpha diversity increases on lesional skin after nb-UVB treatment for 6-8 weeks in adults with atopic dermatitis. InvSimpson diversity before and after treatment on lesional skin (LS; $p=0.013$; $\mathbf{a}$ ) and nonlesional skin (NLS; $p=0.305 ; \mathbf{b})$. c Proportions of predominant genera on LS and NLS before and after nb-UVB treatment: Staphylococcus (in yellow). Each column represents 1 sample. The relative abundance of Staphylococcus was higher on LS than NLS for untreated $(p=0.001 ; \mathbf{d})$ and treated skin $(p=0.001 ; \mathbf{e})$.

Shifts in the Skin Microbiota after UVB Treatment in AD
Dermatology 2022;238:109-120

DOI: $10.1159 / 000515236$ 


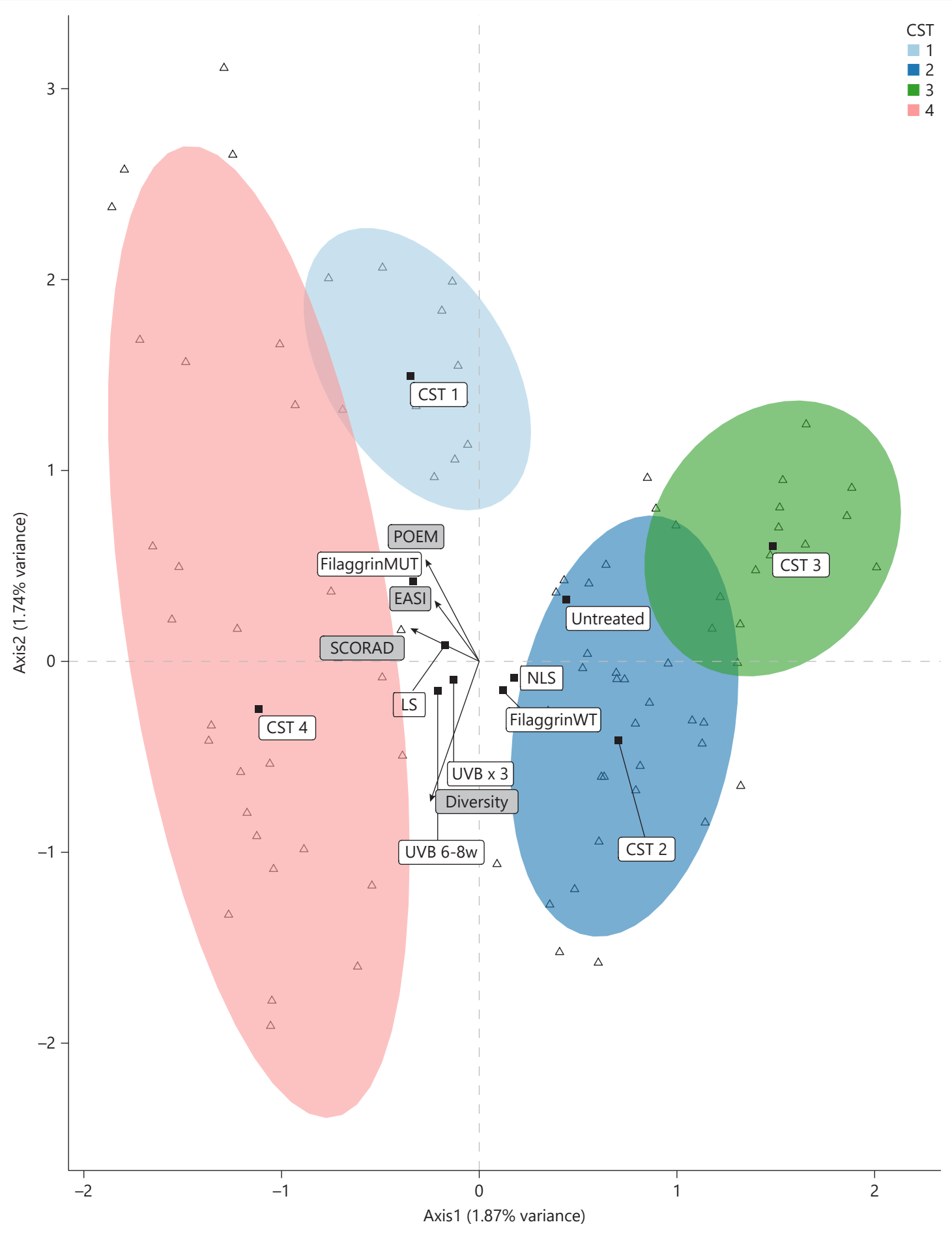

Fig. 3. Canonical correspondence analysis (CCpnA) to model the relationship between community state type (CST) affiliation, bacterial alpha diversity, and clinical severity in adults with atopic dermatitis. The colored ellipses represent the CSTs and the samples cluster into 4 distinct CSTs. CSTs 1 and 4 are more common in lesional skin (LS) than in non-lesional skin (NLS). Clinical severity scores are higher in samples grouped into CSTs 1 and 4, and lower in samples grouped into CST 2. Black squares represent centroids of categorical variables (white labels). Arrows represent continuous variables (grey labels). Triangles represent samples. UVB, ultraviolet B; FilaggrinMUT/WT, filaggrin mutation present/wild type; EASI, Eczema Activity and Severity Index; SCORAD, Scoring Atopic Dermatitis index; POEM, Patient-Oriented Eczema Measurement. 
common in LS than in NLS samples. Clinical severity scores were higher in samples grouped into CSTs 1 and 4 and lower in samples grouped into CST 2 (Fig. 3).

Clinical severity scores were higher when an FLG mutation was present (Fig. 3) but due to the small number of patients with a mutation, this association remains inconclusive and must be confirmed in a larger study population. Transition probabilities between CSTs were visualized as Markov chains (online suppl. Fig. S2). CSTs 1 and 4 were the most stable, while CST 2 was more dynamic. Although not statistically significant, we observed a trend towards decreasing clinical severity scores when shifting to CST 2 after nb-UVB treatment compared to switching to or staying in another CST (data not shown).

Nose and Throat Microbiota Are Not Affected by FullBody nb-UVB Treatment

We could not find any changes in alpha diversity in the nose or throat after 6-8 weeks of full-body treatment with nb-UVB (data not shown). The relative abundance on the genus level in the nose is shown in Figure 4a; according to the plots, there were no major alterations before and after treatment. We also tested if the relative abundance of the genus Staphylococcus was altered, but it did not change (Fig. 4b). We could not observe any major changes in the relative abundance on the genus level in the throat either (Fig. 4c).

\section{Short-Term Treatment Does Not Result in Observable Microbiota Shifts}

To shed light on whether nb-UVB could induce early shifts in the skin microbiota before any signs of clinical remission were evident, we looked at alpha diversity on LS before and after only 3 treatments of local nb-UVB. We did not observe any statistically significant changes in microbial alpha diversity (online suppl. Fig. S3) and the relative abundance of the genus Staphylococcus was not changed (data not shown). Plots of relative abundances on the family or genus level did not reveal any major alterations (data not shown).

\section{Discussion}

In this study, we show that full-body nb-UVB treatment for 6-8 weeks changes the composition of the microbiota on LS, but not on NLS, in AD. We have previously shown that gene expression changes are evident in AD skin after only 3 treatments of nb-UVB; specifically, we found altered gene expression of several AMPs and transcripts related to inflammation, epidermal structure, and keratinization [39]. In contrast, we found no significant changes in the skin microbiota after 3 treatments of $\mathrm{nb}-\mathrm{UVB}$. Taken together, these findings may suggest that the changes in the microbiota are secondary to skin inflammation and not a primary driver of $\mathrm{AD}$ pathogenesis, a question which has been raised by several authors $[2,4,5]$.

Our findings of diversity change during treatment are in line with previous studies where alpha diversity increased with clinical improvement $[15,16,62]$. Conventional culturing methods have shown that UVB re-establishes the skin microbiota in $\mathrm{AD}$ [33-35], but this is the first study using $16 \mathrm{~S}$ rRNA gene sequencing to show that $\mathrm{nb}-\mathrm{UVB}$ alone induced changes in the microbiota. A previous study showed increased alpha diversity after 4-6 weeks of treatment with nb-UVB combined with topical corticosteroids [62], but no significant difference from treatment with corticosteroids alone, implying no additional effect of nb-UVB. In the group treated with combination therapy, however, clinical severity scores (EASI) decreased further 3 weeks after discontinuing treatment, indicating a possible positive long-term effect of nb-UVB [62].

Topical corticosteroid treatment for 4 weeks has also been shown to increase the alpha diversity in children with $\mathrm{AD}$ [15], but a shorter treatment duration (7-10 days) did not have any effect on bacterial diversity in infants [63]. A study on dupilumab (a monoclonal antibody blocking interleukin [IL]-4 and IL-13) in adult AD patients showed increased alpha diversity and a lower abundance of S. aureus on LS and NLS after 16 weeks of treatment, but the effect did not last; 18 weeks after discontinuation of treatment, the microbiota closely resembled the pre-treatment state [21].

The effect of UVB is not restricted to $\mathrm{AD}$, as a recent study in healthy subjects $(n=6)$ showed that both UVA and UVB influence the composition of the skin microbiota [44]. In this study on healthy skin, the relative abundance of Pseudomonadaceae decreased after nbUVB exposure [44], but we could not replicate this finding in our material. This could possibly reflect the differences in the skin microbiota in healthy and atopic skin. In psoriasis, another chronic inflammatory skin disease, nb-UVB treatment induces no significant alterations in alpha or beta diversity but significant changes on the genus level [64].

The nasal microbiota in patients with $\mathrm{AD}$ is less diverse than in healthy controls [23]. We hypothesized that full-body nb-UVB treatment might also modulate the na- 


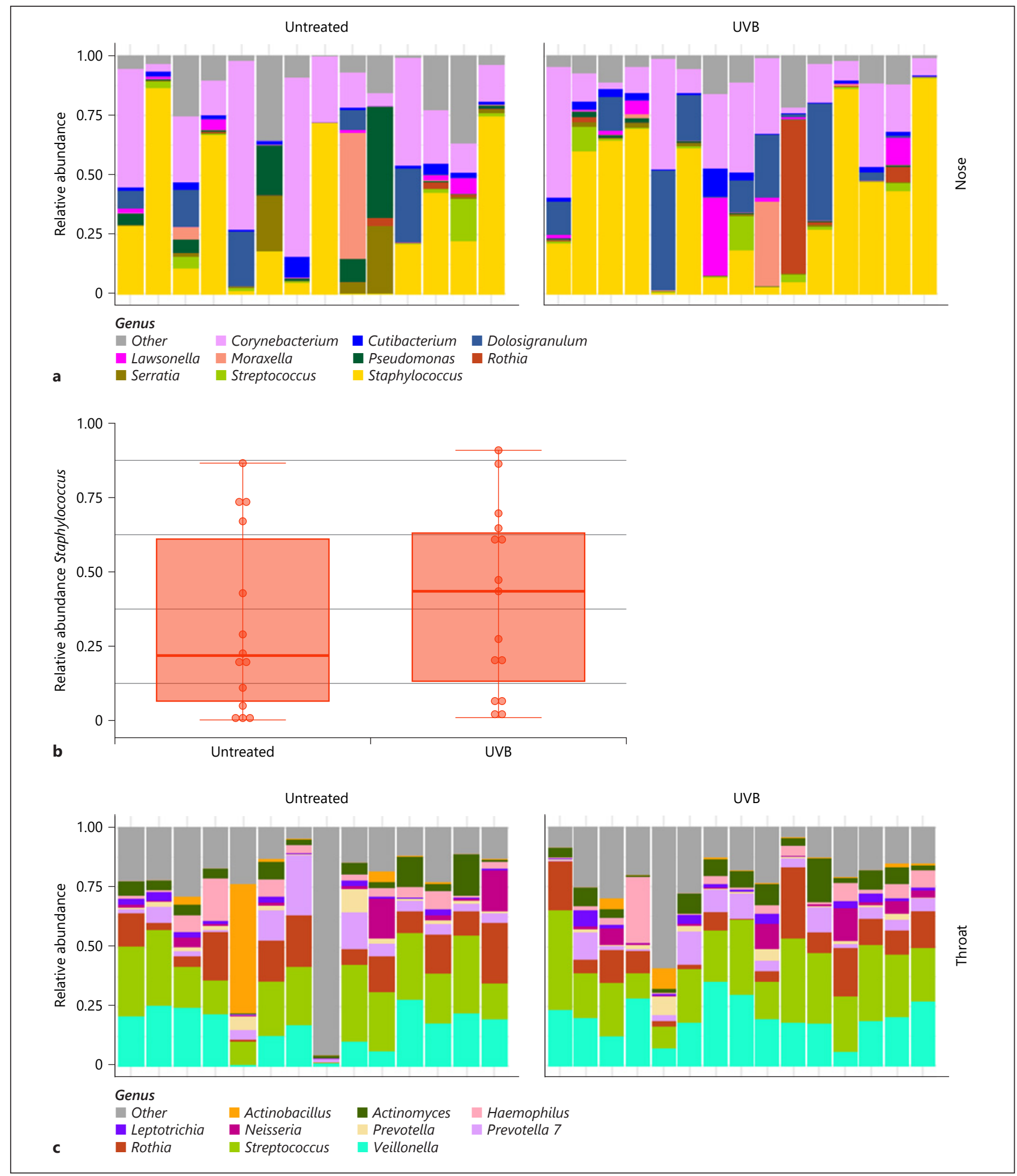

Fig. 4. The microbiome of the nose and throat in adults with atopic dermatitis. a In the nose, there were no changes on the genus level after treatment. b The relative abundance of the genus Staphylococcus did not change. c In the throat, there were no changes after treatment on the genus level. Each column represents 1 sample. 
sal and throat microbiota. However, we found no changes in alpha diversity in the nose or throat after $6-8$ weeks treatment with nb-UVB, suggesting that UVB irradiation of the skin has less effect on the microbiota on mucosal surfaces. We are not aware of any studies having explored the effect of nb-UVB on the nasal or throat microbiota in AD. However, a recent study showed that nb-UVB irradiation of the skin in healthy individuals is associated with increased diversity of the gut microbiota [65]. This modulation was restricted to individuals not on vitamin D supplements, suggesting that vitamin D levels could influence the microbial composition of the gut. Limited UVB exposure and low vitamin D is thought to partly explain the prevalence of several other chronic inflammatory diseases and the correlation between latitude and disease severity $[66,67]$. In our study, half of the participants took some form of vitamin D supplements (Table 1 ), but we did not stratify on this parameter in our analyses. Serum vitamin D levels increased in our cohort, confirming previous reports [68-70].

Our patient cohort is well-characterized, and disease severity is scored by validated scoring tools in line with guidelines from the Harmonizing Outcome Measuring Eczema (HOME) initiative, in order to make different studies easier to compare [71]. The patient population seems quite representative of adult AD patients; approximately $25 \%$ had an FLG mutation which corresponds to previously reported numbers [72]. Most of the patients in our cohort responded well to treatment with nb-UVB [39], consistent with previous reports on nb-UVB treatment $[73,74]$.

The main limitation of our study is the small sample size, which rendered it mainly explorative; further studies with more participants are needed to validate our results. Although16S rRNA gene sequencing provides extensive information about microbial communities on the skin [37], the copy number of the 16S rRNA gene varies between bacterial species and may lead to an over-representation of some species [75]. Furthermore, 16S rRNA gene sequencing results are compositional rather than absolute, and our analysis did not determine any cause-andeffect relationships [36]. Taxonomy is dependent on the reference database used [36] and technical aspects like sampling technique, DNA extraction, and sequencing protocol may introduce some degree of uncertainty, especially between studies [76, 77]. To introduce as little variation as possible, all samples in this study were processed and treated the same way.

Despite the obvious dysbiosis in the eczematous skin of patients with $\mathrm{AD}$, treatments modulating the micro- biota have not been central in clinical practice. Two small-scale trials reintroduced commensal skin bacteria in human subjects with AD. Topical application of coagulase-negative Staphylococcus spp. decreased the colonization by $S$. aureus [78], and topical application of the Gram-negative Roseomonas mucosa led to clinical improvement [79]. More knowledge regarding the complex interplay between the skin microbiota and the immune system could pave the way for more individualized treatment.

Treatment with nb-UVB for $6-8$ weeks in adults with $\mathrm{AD}$ induced shifts in the skin microbiota in LS, but did not affect the microbiota of NLS, or the nose and throat. The changes in LS appeared after clinical remission was evident, suggesting that the microbial changes are secondary, rather than primary, in the pathogenesis of $\mathrm{AD}$.

\section{Key Message}

UVB treatment for 6-8 weeks increases bacterial diversity in lesional skin in atopic dermatitis.

\section{Appendix 1}

Truncation Length Adjustments

For 16S rRNA reads, truncation lengths were adjusted to 270 bp for forward reads and $210 \mathrm{bp}$ for reverse reads. For $t u f$ gene reads, forward reads were truncated at $270 \mathrm{bp}$ and reverse reads were truncated at $241 \mathrm{bp}$.

\section{Appendix 2}

Contaminant Removal

Contaminants were identified and removed manually: ASVs classified no further than class-level and belonging to either the phyla Cyanobacteria, Plantomycetes, Chloroflexi, and Deinococcus-Thermus, or the orders Rhizobiales, Rhodobacterales, and Oceanospirillales. From the skin sample data set, 38 ASVs were identified as contaminants and removed (decontam method "either", frequency threshold 0.1, prevalence threshold 0.25). In addition, 224 ASVs were removed manually. From the nasal data set, we removed 27 ASVs (decontam method "either", frequency threshold 0.05, prevalence threshold 0.25), and 89 ASVs were removed manually. We removed 47 ASVs from the throat data set (decontam method "either", frequency threshold 0.1, prevalence threshold 0.25). Twenty-five ASVs were manually removed. After contaminant removal, read counts of re-sequenced samples were merged. 


\section{Acknowledgements}

We would like to thank the patients who participated in the study. We would also like to thank PEF, NAAF, the Arne E. Ingel's endowment, and Sanofi for financial support.

\section{Statement of Ethics}

The Regional Ethics Committee approved the study (2017/466). All participants gave informed written consent.

\section{Conflict of Interest Statement}

AHL has received an unrestricted research grant from Sanofi.

\section{Funding Sources}

This work was supported by the University of Oslo and Oslo University Hospital, research grants from patient organizations (The Psoriasis and Eczema Foundation [PEF], The Norwegian Asthma and Allergy Foundation [NAAF]), the Arne E. Ingel's endowment, and a research grant from Sanofi. None of these sources had any role in study design, data collection, data analysis, manuscript preparation or publication decisions.

\section{Author Contributions}

A.H.L., T.L.B., J.-Ø.H., O.S., and G.H. designed the study. A.H.L. included the patients. J.V.B. handled the samples. S.M.E., A.C.I., B.L., and P.S.-A. sequenced and analyzed the samples. S.A. and M.B. did the filaggrin analyses. A.H.L. drafted the manuscript and all co-authors contributed to the editing process.

\section{References}

1 Deckers IA, McLean S, Linssen S, Mommers $\mathrm{M}$, van Schayck CP, Sheikh A. Investigating international time trends in the incidence and prevalence of atopic eczema 1990-2010: a systematic review of epidemiological studies. PLoS One. 2012;7(7):e39803.

2 Bieber T. Atopic dermatitis. Ann Dermatol. 2010 May;22(2):125-37.

3 Alexander H, Paller AS, Traidl-Hoffmann C, Beck LA, De Benedetto A, Dhar S, et al. The role of bacterial skin infections in atopic dermatitis: expert statement and review from the International Eczema Council Skin Infection Group. Br J Dermatol. 2020 Jun; 182(6):133142.

4 Weidinger S, Beck LA, Bieber T, Kabashima $\mathrm{K}$, Irvine AD. Atopic dermatitis. Nat Rev Dis Primers. 2018 Jun;4(1):1.

5 Langan SM, Irvine AD, Weidinger S. Atopic dermatitis. Lancet. 2020 Aug;396(10247): 345-60.

6 Leyden JJ, Marples RR, Kligman AM. Staphylococcus aureus in the lesions of atopic dermatitis. Br J Dermatol. 1974 May;90(5):52530.

7 Totté JE, van der Feltz WT, Hennekam M, van Belkum A, van Zuuren EJ, Pasmans SG. Prevalence and odds of Staphylococcus aureus carriage in atopic dermatitis: a systematic review and meta-analysis. Br J Dermatol. 2016 Oct;175(4):687-95.

8 Tauber M, Balica S, Hsu CY, Jean-Decoster C, Lauze C, Redoules D, et al. Staphylococcus aureus density on lesional and nonlesional skin is strongly associated with disease severity in atopic dermatitis. J Allergy Clin Immunol. 2016 Apr;137(4):1272-4.e3.

9 Paller AS, Kong HH, Seed P, Naik S, Scharschmidt TC, Gallo RL, et al. The microbiome in patients with atopic dermatitis. J Allergy Clin Immunol. 2019 Jan;143(1):26-35.
10 Miajlovic H, Fallon PG, Irvine AD, Foster TJ. Effect of filaggrin breakdown products on growth of and protein expression by Staphylococcus aureus. J Allergy Clin Immunol. 2010 Dec;126(6):1184-90.e3.

11 Bjerre RD, Bandier J, Skov L, Engstrand L, Johansen JD. The role of the skin microbiome in atopic dermatitis: a systematic review. $\mathrm{Br} \mathrm{J}$ Dermatol. 2017 Nov;177(5):1272-8.

12 Shi B, Bangayan NJ, Curd E, Taylor PA, Gallo RL, Leung DY, et al. The skin microbiome is different in pediatric versus adult atopic dermatitis. J Allergy Clin Immunol. 2016 Oct; 138(4):1233-6.

13 Salava A, Lauerma A. Role of the skin microbiome in atopic dermatitis. Clin Transl Allergy. 2014 Oct;4(1):33.

14 Bourrain M, Ribet V, Calvez A, Lebaron P, Schmitt AM. Balance between beneficial microflora and Staphylococcus aureus colonisation: in vivo evaluation in patients with atopic dermatitis during hydrotherapy. Eur J Dermatol. 2013 Nov-Dec;23(6):786-94.

15 Gonzalez ME, Schaffer JV, Orlow SJ, Gao Z, Li H, Alekseyenko AV, et al. Cutaneous microbiome effects of fluticasone propionate cream and adjunctive bleach baths in childhood atopic dermatitis. J Am Acad Dermatol. 2016 Sep;75(3):481-93.e8.

16 Kong HH, Oh J, Deming C, Conlan S, Grice EA, Beatson MA, et al.; NISC Comparative Sequence Program. Temporal shifts in the skin microbiome associated with disease flares and treatment in children with atopic dermatitis. Genome Res. 2012 May;22(5): 850-9.

17 Byrd AL, Deming C, Cassidy SK, Harrison OJ, Ng WI, Conlan S, et al.; NISC Comparative Sequencing Program. Staphylococcus aureus and Staphylococcus epidermidis strain diversity underlying pediatric atopic dermatitis. Sci Transl Med. 2017 Jul;9(397):eaal4651.
18 Seite S, Flores GE, Henley JB, Martin R, Zelenkova H, Aguilar L, et al. Microbiome of affected and unaffected skin of patients with atopic dermatitis before and after emollient treatment. J Drugs Dermatol. 2014 Nov; 13(11):1365-72.

19 Clausen ML, Edslev SM, Nørreslet LB, Sørensen JA, Andersen PS, Agner T. Temporal variation of Staphylococcus aureus clonal complexes in atopic dermatitis: a follow-up study. Br J Dermatol. 2019 Jan;180(1):181-6.

20 Edslev SM, Agner T, Andersen PS. Skin Microbiome in Atopic Dermatitis. Acta Derm Venereol. 2020 Jun;100(12):adv00164.

21 Callewaert C, Nakatsuji T, Knight R, Kosciolek T, Vrbanac A, Kotol P, et al. IL-4Ra Blockade by Dupilumab Decreases Staphylococcus aureus Colonization and Increases Microbial Diversity in Atopic Dermatitis. J Invest Dermatol. 2020 Jan;140(1):191-202.e7.

22 Baurecht $\mathrm{H}$, Rühlemann MC, Rodríguez E, Thielking F, Harder I, Erkens AS, et al. Epidermal lipid composition, barrier integrity, and eczematous inflammation are associated with skin microbiome configuration. J Allergy Clin Immunol. 2018 May;141(5):1668-76.e16.

23 Clausen ML, Agner T, Lilje B, Edslev SM, Johannesen TB, Andersen PS. Association of Disease Severity With Skin Microbiome and Filaggrin Gene Mutations in Adult Atopic Dermatitis. JAMA Dermatol. 2018 Mar; 154(3):293-300.

24 Brandwein M, Fuks G, Israel A, Sabbah F, Hodak E, Szitenberg A, et al. Skin Microbiome Compositional Changes in Atopic Dermatitis Accompany Dead Sea Climatotherapy. Photochem Photobiol. 2019 Nov;95(6):1446-53.

25 Totté JE, Pardo LM, Fieten KB, Vos MC, van den Broek TJ, Schuren FH, et al. Nasal and skin microbiomes are associated with disease severity in paediatric atopic dermatitis. $\mathrm{Br}$ Dermatol. 2019 Oct;181(4):796-804. 
26 Lebon A, Labout JA, Verbrugh HA, Jaddoe VW, Hofman A, van Wamel WJ, et al. Role of Staphylococcus aureus nasal colonization in atopic dermatitis in infants: the Generation $\mathrm{R}$ Study. Arch Pediatr Adolesc Med. 2009 Aug; 163(8):745-9.

27 Yoshimura M, Namura S, Akamatsu H, Horio T. Antimicrobial effects of phototherapy and photochemotherapy in vivo and in vitro. $\mathrm{Br} \mathrm{J}$ Dermatol. 1996 Oct;135(4):528-32.

28 Yoshimura-Mishima M, Akamatsu H, Namura S, Horio T. Suppressive effect of ultraviolet (UVB and PUVA) radiation on superantigen production by Staphylococcus aureus. J Dermatol Sci. 1999 Jan;19(1):31-6.

29 Faergemann J, Larkö O. The effect of UVlight on human skin microorganisms. Acta Derm Venereol. 1987;67(1):69-72.

30 Gambichler T, Skrygan M, Tomi NS, Altmeyer P, Kreuter A. Changes of antimicrobial peptide mRNA expression in atopic eczema following phototherapy. Br J Dermatol. 2006 Dec;155(6):1275-8.

31 Hong SP, Kim MJ, Jung MY, Jeon H, Goo J, Ahn SK, et al. Biopositive effects of low-dose UVB on epidermis: coordinate upregulation of antimicrobial peptides and permeability barrier reinforcement. J Invest Dermatol. 2008 Dec;128(12):2880-7.

32 Gläser R, Navid F, Schuller W, Jantschitsch C, Harder J, Schröder JM, et al. UV-B radiation induces the expression of antimicrobial peptides in human keratinocytes in vitro and in vivo. J Allergy Clin Immunol. 2009 May; 123(5):1117-23.

33 Dotterud LK, Wilsgaard T, Vorland LH, Falk ES. The effect of UVB radiation on skin microbiota in patients with atopic dermatitis and healthy controls. Int J Circumpolar Health. 2008 Jun;67(2-3):254-60.

34 Jekler J, Bergbrant IM, Faergemann J, Larkö $\mathrm{O}$. The in vivo effect of $\mathrm{UVB}$ radiation on skin bacteria in patients with atopic dermatitis. Acta Derm Venereol. 1992;72(1):33-6.

35 Silva SH, Guedes AC, Gontijo B, Ramos AM, Carmo LS, Farias LM, et al. Influence of narrow-band UVB phototherapy on cutaneous microbiota of children with atopic dermatitis. J Eur Acad Dermatol Venereol. 2006 Oct; 20(9):1114-20.

36 Jo JH, Kennedy EA, Kong HH. Research Techniques Made Simple: Bacterial 16S Ribosomal RNA Gene Sequencing in Cutaneous Research. J Invest Dermatol. 2016 Mar; 136(3):e23-7.

37 Grogan MD, Bartow-McKenney C, Flowers L, Knight SAB, Uberoi A, Grice EA. Research Techniques Made Simple: Profiling the Skin Microbiota. J Invest Dermatol. 2019;139(4): 747-52.e1.

38 Hanifin JM, Rajka G. Diagnostic features of atopic dermatitis. Acta Dermatovener (Stockholm) Suppl. 1980;60:44-7.
39 Lossius AH, Berents TL, Saetre F, Nilsen HR, Bradley M, Asad S, et al. Early transcriptional changes after UVB treatment in atopic dermatitis includes inverse regulation of IL-36y and IL-37. Exp Dermatol. 2020, Online ahead of print.

40 Hanifin JM, Thurston M, Omoto M, Cherill R, Tofte SJ, Graeber M, et al.; EASI Evaluator Group. The eczema area and severity index (EASI): assessment of reliability in atopic dermatitis. Exp Dermatol. 2001 Feb;10(1):11-8.

41 Kunz B, Oranje AP, Labrèze L, Stalder JF, Ring J, Taïeb A. Clinical validation and guidelines for the SCORAD index: consensus report of the European Task Force on Atopic Dermatitis. Dermatology. 1997;195(1):10-9.

42 Charman CR, Venn AJ, Williams HC. The patient-oriented eczema measure: development and initial validation of a new tool for measuring atopic eczema severity from the patients' perspective. Arch Dermatol. 2004 Dec;140(12):1513-9.

43 Finlay AY, Khan GK. Dermatology Life Quality Index (DLQI) - a simple practical measure for routine clinical use. Clin Exp Dermatol. 1994 May; 19(3):210-6.

44 Burns EM, Ahmed H, Isedeh PN, Kohli I, Van Der Pol W, Shaheen A, et al. Ultraviolet radiation, both UVA and UVB, influences the composition of the skin microbiome. Exp Dermatol. 2019 Feb;28(2):136-41.

45 Klindworth A, Pruesse E, Schweer T, Peplies J, Quast C, Horn M, et al. Evaluation of general 16S ribosomal RNA gene PCR primers for classical and next-generation sequencingbased diversity studies. Nucleic Acids Res. 2013 Jan;41(1):e1.

46 Iversen S, Johannesen TB, Ingham AC, Edslev SM, Tevell S, Månsson E, et al. Alteration of Bacterial Communities in Anterior Nares and Skin Sites of Patients Undergoing Arthroplasty Surgery: Analysis by $16 \mathrm{~S}$ rRNA and Staphylococcal-Specific tuf Gene Sequencing. Microorganisms. 2020 Dec;8(12):1977.

47 Martin M. CUTADAPT removes adapter sequences from high-throughput sequencing reads. EMBnet J. 2011;17(1):17.

48 Callahan BJ, McMurdie PJ, Rosen MJ, Han AW, Johnson AJ, Holmes SP. DADA2: highresolution sample inference from Illumina amplicon data. Nat Methods. 2016 Jul;13(7): 581-3.

49 Callahan BJ. Silva taxonomic training data formatted for DADA2 (Silva version 132) 2018.

50 McMurdie PJ, Holmes S. phyloseq: an R package for reproducible interactive analysis and graphics of microbiome census data. PLoS One. 2013 Apr;8(4):e61217.

51 Davis NM, Proctor DM, Holmes SP, Relman DA, Callahan BJ. Simple statistical identification and removal of contaminant sequences in marker-gene and metagenomics data. Microbiome. 2018 Dec;6(1):226.
52 R_Core_Team. R: A Language and Environment for Statistical Computing.: R Foundation for Statistical Computing, Vienna, Austria.; 2019. Available from: https://www.Rproject.org/.

53 Oksanen J, Blanchet FG, Friendly M, Kindt R, Legendre P, McGlinn D, et al. vegan: Community Ecology Package. R package version 2.5-5. 2019. Available from: https://CRAN.Rproject.org $/$ package $=$ vegan

54 Maechler M, Rousseeuw P, Struyf A, Hubert M, Hornik K. cluster: Cluster Analysis Basic and Extensions. R package version 2.1.0 2019.

55 Kassambara A, Mundt F. factoextra: Extract and Visualize the Results of Multivariate Data Analyses. R package version 1.0.6 2019. Available from: https://CRAN.R-project.org/ package $=$ factoextra.

56 Signorell A. DescTools: Tools for descriptive statistics. R package version 0.99.31 2019.

57 Kuznetsova A, Brockhoff PB, Christensen RHB. lmerTest Package: Tests in Linear Mixed Effects Models. J Stat Softw. 2017; 82(13):26.

58 Spedicato G. Discrete Time markov Chains with R. R J. 2017;9(2):84.

$59 \mathrm{Gu}$ Z, Eils R, Schlesner M. Complex heatmaps reveal patterns and correlations in multidimensional genomic data. Bioinformatics. 2016 Sep;32(18):2847-9.

60 Wickham H. ggplot2: Elegant Graphics for Data Analysis. 2nd ed: Springer Verlag New York; 2016

61 Soetaert K. diagram: Functions for Visualizing Simple Graphs (Networks), Plotting Flow Diagrams. R package 1.6.4. 2017. Available from: package $=$ diagram

62 Kwon S, Choi JY, Shin JW, Huh CH, Park KC, $\mathrm{Du} \mathrm{MH}$, et al. Changes in Lesional and Nonlesional Skin Microbiome During Treatment of Atopic Dermatitis. Acta Derm Venereol. 2019 Mar;99(3):284-90.

63 Zheng Y, Wang Q, Ma L, Chen Y, Gao Y, Zhang G, et al. Alterations in the skin microbiome are associated with disease severity and treatment in the perioral zone of the skin of infants with atopic dermatitis. Eur J Clin Microbiol Infect Dis. 2019 Sep;38(9):1677-85.

64 Assarsson M, Duvetorp A, Dienus O, Söderman J, Seifert O. Significant Changes in the Skin Microbiome in Patients with Chronic Plaque Psoriasis after Treatment with Narrowband Ultraviolet B. Acta Derm Venereol. 2018 Apr;98(4):428-36.

65 Bosman ES, Albert AY, Lui H, Dutz JP, Vallance BA. Skin Exposure to Narrow Band Ultraviolet (UVB) Light Modulates the Human Intestinal Microbiome. Front Microbiol. 2019 Oct; $10: 2410$ 
$66 \mathrm{Lu}$ C, Yang J, Yu W, Li D, Xiang Z, Lin Y, et al. Association between 25(OH)D Level, Ultraviolet Exposure, Geographical Location, and Inflammatory Bowel Disease Activity: A Systematic Review and Meta-Analysis. PLoS One. 2015 Jul;10(7):e0132036.

67 Breuer J, Schwab N, Schneider-Hohendorf T, Marziniak M, Mohan H, Bhatia U, et al. Ultraviolet B light attenuates the systemic immune response in central nervous system autoimmunity. Ann Neurol. 2014 May;75(5): 739-58.

68 Le P, Tu J, Gebauer K, Brown S. Serum 25-hydroxyvitamin D increases with NB-UVB and UVA/UVB phototherapy in patients with psoriasis and atopic dermatitis in Western Australia. Australas J Dermatol. 2016 May; 57(2):115-21.

69 Cicarma E, Mørk C, Porojnicu AC, Juzeniene A, Tam TT, Dahlback A, et al. Influence of narrowband UVB phototherapy on vitamin D and folate status. Exp Dermatol. 2010 Aug; 19(8):e67-72.

70 Bogh MK, Gullstrand J, Svensson A, Ljunggren B, Dorkhan M. Narrowband ultraviolet $B$ three times per week is more effective in treating vitamin D deficiency than $1600 \mathrm{IU}$ oral vitamin $\mathrm{D}_{3}$ per day: a randomized clinical trial. Br J Dermatol. 2012 Sep;167(3):625-30.
71 Schmitt J, Spuls P, Boers M, Thomas K, Chalmers J, Roekevisch E, et al. Towards global consensus on outcome measures for atopic eczema research: results of the HOME II meeting. Allergy. 2012 Sep;67(9):1111-7.

72 Palmer CN, Irvine AD, Terron-Kwiatkowski A, Zhao Y, Liao H, Lee SP, et al. Common loss-of-function variants of the epidermal barrier protein filaggrin are a major predisposing factor for atopic dermatitis. Nat Genet. 2006 Apr;38(4):441-6.

73 Majoie IM, Oldhoff JM, van Weelden $H$, Laaper-Ertmann M, Bousema MT, Sigurdsson V, et al. Narrowband ultraviolet B and medium-dose ultraviolet $\mathrm{A} 1$ are equally effective in the treatment of moderate to severe atopic dermatitis. J Am Acad Dermatol. 2009 Jan;60(1):77-84.

74 Garritsen FM, Brouwer MW, Limpens J, Spuls PI. Photo(chemo)therapy in the management of atopic dermatitis: an updated systematic review with implications for practice and research. Br J Dermatol. 2014 Mar;170(3): 501-13.
75 Pei AY, Oberdorf WE, Nossa CW, Agarwal A, Chokshi P, Gerz EA, et al. Diversity of $16 S$ rRNA genes within individual prokaryotic genomes. Appl Environ Microbiol. 2010 Jun; 76(12):3886-97.

76 Meisel JS, Hannigan GD, Tyldsley AS, SanMiguel AJ, Hodkinson BP, Zheng Q, et al. Skin Microbiome Surveys Are Strongly Influenced by Experimental Design. J Invest Dermatol. 2016 May;136(5):947-56.

77 Kong HH, Andersson B, Clavel T, Common JE, Jackson SA, Olson ND, et al. Performing Skin Microbiome Research: A Method to the Madness. J Invest Dermatol. 2017 Mar; 137(3):561-8.

78 Nakatsuji T, Chen TH, Narala S, Chun KA, Two AM, Yun T, et al. Antimicrobials from human skin commensal bacteria protect against Staphylococcus aureus and are deficient in atopic dermatitis. Sci Transl Med. 2017 Feb;9(378):eaah4680.

79 Myles IA, Earland NJ, Anderson ED, Moore IN, Kieh MD, Williams KW, et al. First-inhuman topical microbiome transplantation with Roseomonas mucosa for atopic dermatitis. JCI Insight. 2018 May;3(9):120608. 